\title{
9. A SERIES-SOLUTION METHOD FOR COMETARY ORBITS
}

\author{
P. E. NACOZY \\ Dept. of Aerospace Engineering and Engineering Mechanics, \\ University of Texas, Austin, Tex., U.S.A.
}

\begin{abstract}
A series-solution method for highly-eccentric perturbed orbits using a modified form of Hansen's method of partial anomalies is presented. Series in Chebyshev polynomials in the eccentric anomaly of a comet and the mean anomaly at an epoch of a planet provide a theory valid to first order with respect to the masses. The first-order solution becomes a reference solution about which higher-order perturbations are obtained by the method of successive approximations. The first-order solutions are valid approximations for long durations of time, whereas the higher orders are valid only over the interval of time that is selected for the Chebyshev expansions. The method is somewhat similar to Encke's method of special perturbations except that for each successive interval of time perturbations about a first-order solution are calculated instead of perturbations about a conic solution.
\end{abstract}

\section{Introduction}

The application of conventional methods of general perturbations to the highly eccentric orbits of comets and certain asteroids presents several difficulties. Two of the most severe difficulties are the slowness of convergence of the series-solutions, if they converge at all, and the inability of low-order solutions to be valid over long intervals of time.

The convergence of the developments may be strengthened by expansions in the eccentric anomaly of the comet and by segmentation of the reference orbit of the comet following the ideas of Hansen (1856) and Gyldén (1870). But, for accuracy over longer intervals of time, higher-order solutions must be obtained.

The present paper discusses a method of general perturbations that utilizes expansions in Chebyshev polynomials in the eccentric anomaly and segmentation of the orbit to strengthen convergence. The method incorporates the Picard method of successive approximations to attain a high-order solution.

The use of Chebyshev polynomials and Picard's method for the solution of nonlinear differential equations in initial and boundary value problems has been well studied (see, for example, Clenshaw and Norton, 1963; Fox and Parker, 1968). The technique has been applied to the planetary system, with notable success, by Carpenter (1966) and Broucke (1969).

This paper presents a method that yields series-solutions of cometary orbits in double Chebyshev series and utilizes a reference solution comprising both the zerothorder (conic motion) and the first-order solution (with respect to the disturbing masses). This reference solution approximates more closely the true solution than does a conic reference solution. The method allows the perturbing planets to have their actual nonelliptical motion. The method is semianalytical in that the series have numerical coefficients. 


\section{Expansion in Chebyshev Polynomials}

The method of general perturbations presented here may be applied to any formulation of the differential equations of motion of a comet. For this discussion, we will define the perturbations by second-order differential equations in rectangular coordinates. For most other equations and coordinates, all of the following will apply with minor modifications. Let

$$
\mathbf{r}=\mathbf{r}_{0}+\delta \mathbf{r},
$$

where $\mathbf{r}$ is the actual position vector to the comet, $\mathbf{r}_{0}$ the position vector to the reference conic, and $\delta \mathbf{r}$ the perturbation of the comet. Let $\mathbf{r}_{j}^{\prime}$ be the position vector to the $j$ th perturbing planet and allow $p$ perturbing planets. The heliocentric perturbation equations may be simply written as

$$
\begin{aligned}
\frac{d^{2}}{d t^{2}} \delta \mathbf{r} & =-\mu\left(\frac{\mathbf{r}}{|\mathbf{r}|^{3}}-\frac{\mathbf{r}_{0}}{\left|\mathbf{r}_{0}\right|^{3}}\right)+\mu \sum_{j=1}^{p} m_{j}^{\prime}\left(\frac{\mathbf{r}_{j}^{\prime}-\mathbf{r}}{\left|\mathbf{r}_{j}^{\prime}-\mathbf{r}\right|^{3}}-\frac{\mathbf{r}_{j}^{\prime}}{\left|\mathbf{r}_{j}^{\prime}\right|^{3}}\right) \\
& =\varphi\left(\mathbf{r}_{0}, \mathbf{r} ; \mathbf{r}_{1}^{\prime}, \mathbf{r}_{2}^{\prime}, \ldots, \mathbf{r}_{p}^{\prime}\right) .
\end{aligned}
$$

In Equation (1), $\mu$ is the gravitational constant and $m_{j}^{\prime}$ the mass of the $j$ th perturbing planet, and $\varphi$ is a vector function. Let us confine the following discussion to the presence of only one perturbing planet. The generalization to $p$ perturbing planets is straightforward.

Let us transform the independent variable of Equation (1) to the eccentric anomaly $u$ of the reference conic using the relation

$$
\mathrm{d} t=\left(r_{0} / n_{0} a_{0}\right) \mathrm{d} u,
$$

where $r_{0}=\left|\mathbf{r}_{0}\right|$, and $n_{0}$ and $a_{0}$ are the mean motion and semimajor axis of the reference conic, respectively. The function $\varphi$ of Equation (1) is also transformed through Kepler's equation to a function of the eccentric anomaly $u$ of the comet, as will be shown later. Let us proceed formally to integrate Equation (1) by the Picard method of successive approximations. Let the subscript $n$ denote the $n$th iterate. Let $\{\mathbf{r}\}_{n+1}$ denote the position vector determined by the $n$th iterate. Define

$$
\{\boldsymbol{\varphi}\}_{n} \equiv \boldsymbol{\varphi}\left(\mathbf{r}_{0},\{\mathbf{r}\}_{n} ; \mathbf{r}^{\prime}\right)
$$

and

$$
\boldsymbol{\varphi}_{n} \equiv r_{0} \times\{\boldsymbol{\varphi}\}_{n}=\boldsymbol{\varphi}_{n}\left(\mathbf{r}_{0},\{\mathbf{r}\}_{n} ; \mathbf{r}^{\prime}\right) .
$$

Then, using Equations (1), (2), (3a), and (3b), the position vector $\{\mathbf{r}\}_{n+1}$ is given by

$$
\begin{aligned}
& \{\delta \mathbf{r}\}_{n}=\frac{1}{n_{0}^{2} a_{0}^{2}} \int^{u} r_{0} \mathrm{~d} u^{*} \int^{u^{*}} \boldsymbol{\varphi}_{n} \mathrm{~d} u^{*}+\mathbf{a}_{n} t+\mathbf{b}_{n}, \\
& \{\mathbf{r}\}_{n+1}=\mathbf{r}_{0}+\{\delta \mathbf{r}\}_{n} ; \quad n=0,1,2, \cdots .
\end{aligned}
$$

If the function $\varphi$ is continuous and satisfies the Lipschitz condition in a domain of $u$, or, from Equation (1), if neither orbital intersections nor collisions occur between 
comet and planet in an interval of time, then the process defined by Equations (3) is convergent to the true solution of Equation (1) in this interval (see Ince, 1956, pp. 62-92). In Equation (3c), the symbols $\mathbf{a}_{n}$ and $\mathbf{b}_{n}$ are vector constants of integration determined during the $n$th iterate. They will also converge to the true constants of integration satisfying the desired set of boundary conditions.

If we restrict our discussion for the moment to the zeroth iterate of the process (3), we have, since $\{\mathbf{r}\}_{0}=\mathbf{r}_{0}$,

$$
\varphi_{0}=\varphi_{0}\left(\mathbf{r}_{0} ; \mathbf{r}^{\prime}\right) \text {. }
$$

Equations (3) and (4) define a quantity $\{\mathbf{r}\}_{1}$, which is the first-order solution with respect to the masses. With the assumption, which will be removed later, that the perturbing planet moves on a conic, the functions $\mathbf{r}_{0}$ and $\mathbf{r}^{\prime}$ are periodic in the eccentric anomaly of the comet $u$, and the mean anomaly of the planet $l^{\prime}$, respectively, both in $2 \pi$. Hence, the function $\varphi_{0}$, defined by Equation (4), may be represented by a Fourier series with arguments $u$ and $l^{\prime}$. The series may be written as

$$
\boldsymbol{\varphi}_{0}=\sum_{i=0}^{\infty}\left[\sum_{j=0}^{\infty} \alpha_{i j} \sin (j u)\right] \cos \sin \left(i l^{\prime}\right) .
$$

The notation $\begin{aligned} & \sin \\ & \text { cos }\end{aligned}$ denotes a sine and cosine Fourier series. The coefficients, here and in the following series, are vector quantities. Let

$$
l^{\prime}=\frac{n^{\prime}}{n_{0}}\left(u-e_{0} \sin u-c\right)+c^{\prime},
$$

where $n^{\prime}$ is the mean motion of the planet, and $c$ and $c^{\prime}$ define the mean anomalies of the comet and planet, respectively, at an epoch. If we choose the epoch at a perihelion passage of the comet, then, $c=0^{\circ}$ and $c^{\prime}$ is equal to the mean anomaly of the planet at this epoch.

Substituting Equation (6) into the series of Equation (5), it can be shown with the use of Bessel functions that Equation (5) may be transformed into

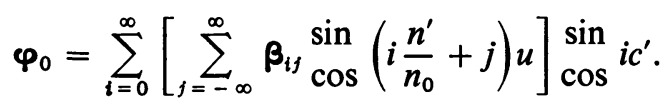

Equations (3c) and (7) yield the first-order perturbation $\{\delta \mathbf{r}\}_{0}$. The position vector $\{\mathbf{r}\}_{1}$ is given by Equation (3d), accurate to first order. Series with the eccentric anomaly of the perturbed body as one argument, as in the series of Equation (7), have often been used in methods of general perturbations to obtain first-order perturbations (see Hansen, 1857; Herget, 1948).

If $n^{\prime} / n_{0}$ is irrational, $\varphi_{0}$, defined by Equation (7), is not periodic in $u$. If $n^{\prime} / n_{0}$ is rational $(=p / q), \varphi_{0}$ is periodic in $u$ with period $2 \pi q$. If $n^{\prime} / n_{0}$ is irrational or if $q$ is large, the coefficients $\beta_{i j}$ or Equation (7) cannot be determined in an efficient manner by harmonic analysis, valid for all $u$. Rather, one must determine first the $\alpha_{i j}$ of Equation (5) and then determine the $\boldsymbol{\beta}_{i j}$ by use of the Bessel functions.

Another difficulty is encountered when we proceed to determine the higher order's 
by the process of Equations (3). The first-order position vector $\{\mathbf{r}\}_{1}$, again assuming no fundamental commensurabilities, is not periodic in $u$, due to the appearance of secular perturbations. Hence, the function $\varphi_{1}$ will not be periodic in $u$, and the coefficients of the Fourier expansion of $\varphi_{1}$ in the arguments $u$ and $l^{\prime}$ cannot be determined efficiently by harmonic analysis, valid for all $u$. This difficulty renders the Picard method of Equation (3) not applicable without some modification.

Both of the above difficulties may be removed by requiring validity of the solution only over a finite interval of the independent variable. With this relaxation, direct harmonic analysis of all orders of the solution is efficient and the method of Picard iteration may be applied. Also, if the perturbing planet cannot be approximated by conic motion, the same may be said of the variable $c^{\prime}$ in the expansion of $\varphi_{1}$.

Any finite interval may be chosen. Hansen, in his memoir on general perturbations for cometary orbits, considering only first-order perturbations, chooses intervals of the independent variable less than one revolution of the comet (Hansen, 1856; Nacozy, 1969). Carpenter (1966), for high-order perturbations of the outer planets, chooses an interval of $200 \mathrm{yr}$. The criterion for the choice of the size of the interval depends mainly on the desired rapidity of convergence of the series.

In the present discussion, we will allow any size interval to be chosen in both variables $u$ and $c^{\prime}$, either less than, equal to, or greater than $2 \pi$ radians. The intervals in $u$ and $c^{\prime}$ will be denoted by $\left[u_{1}, u_{2}\right]$ and $\left[c_{1}^{\prime}, c_{2}^{\prime}\right]$, respectively. It is assumed that $\varphi_{n}$ is not naturally periodic in either $\left[u_{1}, u_{2}\right]$ or $\left[c_{1}^{\prime}, c_{2}^{\prime}\right]$. The Fourier series expansions of $\varphi_{n}$ in the intervals introduces periods $\left[u_{1}, u_{2}\right]$ and $\left[c_{1}^{\prime}, c_{2}^{\prime}\right]$ in $u$ and $c^{\prime}$. Hence, the expansion is not a valid representation for $\boldsymbol{\varphi}_{n}$ outside the chosen intervals $\left[u_{1}, u_{2}\right]$ and $\left[c_{1}^{\prime}, c_{2}^{\prime}\right]$. Also, the induced periodicities require the Fourier expansions to represent discontinuities at the points $u_{1}, u_{2}, c_{1}^{\prime}$ and $c_{2}^{\prime}$. This is undesirable since a discontinuous function has a slowly convergent Fourier series representation. Also, points of discontinuity are inaccurately represented by a truncated Fourier series. An accurate representation of the end points of the solution is extremely important in the continuation of the solution to subsequent (or previous) intervals.

To eliminate the discontinuities, Hansen introduced transformations to new independent variables, which he called the partial anomalies. Identically motivated, we will introduce similar transformations. To remove the discontinuities of the function $\varphi$ at the end points of the interval $\left[u_{1}, u_{2}\right]$ we introduce the transformation

$$
\begin{aligned}
& \frac{2\left(u-u_{1}\right)}{u_{2}-u_{1}}=1+\cos \bar{x}, \\
& -\pi \leq \bar{x} \leq 0, \quad u_{1} \leq u \leq u_{2} .
\end{aligned}
$$

The function $\boldsymbol{\varphi}_{n}, n=0,1,2, \ldots$, no matter what the interval in $u$, becomes a continuous and periodic function of $\bar{x}$. Also, due to the transformation (8), $\varphi_{n}$ becomes an even function of $\bar{x}$. The Fourier expansion of $\varphi_{n}$ in $\bar{x}$, valid only in the interval $\left[u_{1}, u_{2}\right]$, is

$$
\boldsymbol{\varphi}_{n}=\sum_{i=0}^{\infty}\left(\sum_{j=0}^{\infty} \gamma_{i j} \cos j \bar{x}\right) \underset{\cos }{\sin } i c^{\prime}
$$


But $\cos j \bar{x}=T_{j}(x)$, where $x=\cos \bar{x}$ and $T_{j}(x)$ is the Chebyshev polynomial of the $j$ th degree in $x$. The transformation to $x$ is given by

$$
\begin{aligned}
& u=\frac{1}{2}\left(u_{2}-u_{1}\right)(1+x)+u_{1}, \\
& u_{1} \leq u \leq u_{2}, \quad-1 \leq x \leq 1 .
\end{aligned}
$$

The variable $c^{\prime}$ may be transformed similarly. We introduce the transformation

$$
\begin{aligned}
& \frac{2\left(c^{\prime}-c_{1}^{\prime}\right)}{c_{2}^{\prime}-c_{1}^{\prime}}=1+\cos \bar{y}, \\
& -\pi \leq \bar{y} \leq 0, \quad c_{1}^{\prime} \leq c^{\prime} \leq c_{2}^{\prime} .
\end{aligned}
$$

The expansion of the function $\varphi_{n}$, valid in the intervals $\left[u_{1}, u_{2}\right]$ and $\left[c_{1}^{\prime}, c_{2}^{\prime}\right]$, becomes

$$
\boldsymbol{\varphi}_{n}=\sum_{i=0}^{\infty} \sum_{j=0}^{\infty} \boldsymbol{\delta}_{i j} T_{j}(x) \cos i \bar{y} .
$$

But $\cos i \bar{y}=T_{i}(y)$, where $y=\cos \bar{y}$ and $T_{i}(y)$ is the Chebyshev polynomial of the $i$ th degree in $y$. The transformation to $y$ is given by

$$
\begin{aligned}
& c^{\prime}=\frac{1}{2}\left(c_{2}^{\prime}-c_{1}^{\prime}\right)(1+y)+c_{1}^{\prime} \\
& c_{1}^{\prime} \leq c^{\prime} \leq c_{2}^{\prime}, \quad-1 \leq y \leq+1 .
\end{aligned}
$$

The expansion of $\boldsymbol{\varphi}_{n}$ becomes

$$
\begin{aligned}
& \boldsymbol{\varphi}_{n}=\sum_{i=0}^{\infty} \sum_{j=0}^{\infty} \boldsymbol{\delta}_{\imath j} T_{j}(x) T_{i}(y), \\
& -1 \leq x \leq 1, \quad-1 \leq y \leq 1 .
\end{aligned}
$$

The interpretation of the variable $y$ in Equation (10) is as follows. The variable $y$ is related to the variable $c^{\prime}$ through Equation (9b) and $c^{\prime}$ is defined by Equation (6). In Equation (6), we have chosen $c^{\prime}$ to be the value of $l^{\prime}$ at the instant when $u=0$. That is, $c^{\prime}$ is the mean anomaly of the planet at the time of perihelion passage of the comet. With this value for $c^{\prime}$, Equation (6) gives the mean anomaly of the planet $l^{\prime}$ as the comet traverses its reference orbit. Both comet and planet move with mean motions $n_{0}$ and $n^{\prime}$, respectively. With the interval $u_{2}-u_{1}=2 \pi$, introducing Equation (9a) into Equation (6) yields

$$
l^{\prime}=\left(n^{\prime} / n_{0}\right)\left[\pi(1+x)+e_{0} \sin \pi x\right]+c^{\prime} .
$$

Equation (11) gives $l^{\prime}$ only in the interval $c^{\prime} \leq l^{\prime} \leq c^{\prime}+2 \pi\left(n^{\prime} / n_{0}\right)$, for the interval $u_{2}-u_{1}=2 \pi$ and $-1 \leq x \leq 1$. Hence, $c^{\prime}$ is the value of $l^{\prime}$ at the beginning of the chosen interval. For previous or subsequent traversals through the interval, $c^{\prime}$ must be given the value of $l^{\prime}$ at the instant when the comet begins traversing the interval. The corresponding value of $y$ is given by Equation (9b). During each interval $c^{\prime}$ (or $y$ ) remains constant but changes from one interval to the next. If the eccentric anomaly 
of the comet (and hence the mean anomaly) moves through $2 \pi$ radians between successive entries of the interval, then from one traversal to the next,

$$
c^{\prime}=c^{\prime}+2 \pi\left(n^{\prime} / n_{0}\right)
$$

Since the variables $x$ and $y$ are linearly related to the variables $u$ and $c^{\prime}$ by Equations (9), the series (10), truncated to a finite number of terms, is merely a representation of the function $\varphi_{n}$ by a polynomial in $u$ and $c^{\prime}$, valid in the intervals $u_{2}-u_{1}$ and $c_{2}^{\prime}-c_{1}^{\prime}$.

\section{The First-Order Solution}

Consider the intervals $0 \leq u \leq 2 \pi$ and $0 \leq c^{\prime} \leq 2 \pi$. Let $u=0$ correspond to the epoch and let $c^{\prime}$ correspond to the value $l^{\prime}$ at epoch. We are choosing the epoch to be a perihelion passage of the comet and the interval in $u$ to be a revolution of the comet from perihelion to perihelion on the reference conic. The mean anomaly of the perturbing planet $l^{\prime}$ is a function of both $u$ and $c^{\prime}$, or $x$ and $y$ through Equations (9). The function $\varphi_{0}$ may be expanded, by double harmonic analysis, into a truncated, double Chebyshev series in the form of Equation (10) with numerical coefficients. We then proceed with the zeroth iterate of the process of Equations (3). We have

$$
\begin{aligned}
& \boldsymbol{\varphi}_{0}=\sum_{j=0}^{m_{1}} \sum_{i=0}^{m_{2}} \boldsymbol{\delta}_{i j} T_{i}(x) T_{j}(y), \quad \mathrm{d} u=\pi \mathrm{d} x, \\
& \frac{\mathrm{d}}{\mathrm{d} t}\{\delta \mathbf{r}\}_{0}=\frac{1}{n_{0} a_{0}} \int^{u} \boldsymbol{\varphi}_{0} \mathrm{~d} u=\sum_{j=0}^{m_{1}}\left(T_{j}(y) \sum_{i=1}^{m_{2}} \boldsymbol{\varepsilon}_{i j} T_{i}(x)\right)+\mathbf{a}_{0},
\end{aligned}
$$

where

$$
\boldsymbol{\varepsilon}_{i j}=\frac{\pi}{n_{0} a_{0}} \times \frac{1}{2 i}\left(\delta_{i-1, j}-\delta_{i+1, j}\right)
$$

and $\mathbf{a}_{0}$ is a constant of integration. The radius vector of the reference conic is

$$
r_{0}=\left|\mathbf{r}_{0}\right|=a_{0}\left(1-e_{0} \cos u\right)
$$

and may be expanded in a Chebyshev series in $x$. Since the expansion for $r_{0}$ converges faster than the expansion for $\varphi_{0}$, we have $m_{3}<m_{2}$, and

$$
r_{0}=\sum_{\ell=0}^{m_{3}} d_{\ell} T_{\ell}(x) .
$$

From Equation (2), the integral of Equation (12) becomes

$$
\begin{aligned}
\{\delta \mathbf{r}\}_{0}= & \frac{\pi}{n_{0} a_{0}} \sum_{j=0}^{m_{1}} T_{j}(y) \int^{u}\left(\sum_{i=1}^{m_{2}} \boldsymbol{\varepsilon}_{i j} T_{i}(x)\right) \\
& \times\left(\sum_{i=0}^{m_{3}} d_{i} T_{f}(x)\right) \mathrm{d} x+\frac{1}{n_{0}} \mathbf{a}_{0}\left(\pi(x+1)+e_{0} \sin \pi x\right)+\mathbf{b}_{0} .
\end{aligned}
$$


The coefficient of the constant of integration $a_{0}$ in Equation (13) is the time, $t$, in terms of the variable $x$. The integrand of Equation (13), upon multiplication, becomes a series of Chebyshev polynomials of degree $m_{2}+m_{3}=m_{4}$. Let the symbol $\theta_{i j}$ denote the coefficients of the product series. The coefficients $\boldsymbol{\theta}_{i j}$ may be expressed in terms of the coefficients $\boldsymbol{\varepsilon}_{i j}$ and $d_{\ell}$ by straightforward relations. The integral of Equation (13) then becomes

$$
\{\delta \mathbf{r}\}_{0}=\sum_{j=0}^{m_{1}} \sum_{i=1}^{m_{4}} \boldsymbol{\lambda}_{i j} T_{i}(x) T_{j}(y)+\frac{1}{n_{0}} \mathbf{a}_{0}\left(\pi(x+1)+e_{0} \sin \pi x\right)+\mathbf{b}_{0},
$$

where

$$
\lambda_{i j}=\frac{\pi}{n_{0} a_{0}} \times \frac{1}{2 i}\left(\theta_{i-1, j}-\theta_{i+1, j}\right) .
$$

Equation (14) yields the first-order solution for the perturbation $\{\delta \mathbf{r}\}_{0}$. At the epoch, $u=0(x=-1), c^{\prime}$ (or $\left.y\right)$ is equal to the value of $l^{\prime}$ when $u=0$. Denote this value by $y^{0}$. The constants of integration, $\mathbf{a}_{0}$ and $\mathbf{b}_{0}$, are determined so that

$$
\{\delta \mathbf{r}\}_{0}=0, \quad \frac{\mathrm{d}}{\mathrm{d} t}\{\delta \mathbf{r}\}_{0}=0,
$$

at $u=0(x=-1)$. Proceeding to the subsequent (or previous) revolution, the variable $c^{\prime}$ and the constants of integration of the first-order solution of Equation (14) must be reevaluated. For the second evaluation, $c^{\prime}$ (or $y$ ) is equal to the value of $l^{\prime}$ at the instant of time of the subsequent (or previous) perihelion passage of the comet on the reference conic, or at $u=2 \pi$ (or $u=-2 \pi$ ). That is, $c^{\prime}=c^{\prime}+2 \pi\left(n^{\prime} / n_{0}\right)$ at $u=2 \pi$. Denote this new value of $c^{\prime}$ by $y^{1}$. The constants $\mathbf{a}_{0}$ and $\mathbf{b}_{0}$ are determined so that

$$
\left.\{\delta \mathbf{r}\}_{0}\right|_{\substack{x=-1 \\ y=y^{1}}}=\left.\{\delta \mathbf{r}\}_{0}\right|_{\substack{x=+1 \\ y=y^{0}}}
$$

and

$$
\left.\frac{\mathrm{d}}{\mathrm{d} t}\{\delta \mathbf{r}\}_{0}\right|_{\substack{x=-1 \\ y=y^{1}}}=\left.\frac{\mathrm{d}}{\mathrm{d} t}\{\delta \mathbf{r}\}_{0}\right|_{\substack{x=+1 \\ y=y^{0}}} .
$$

And for all previous or subsequent revolutions the determination of $y, \mathbf{a}_{0}$, and $\mathbf{b}_{0}$ proceeds in the same manner. We now possess a reference solution, valid to first order for all time.

\section{The Higher-Order Solution}

We now proceed to obtain a higher-order solution by the Picard method of successive approximations defined by Equations (3). For each revolution or interval in $u$, the successive approximations begin with the first-order solution as the reference solution, not with the zeroth-order solution or reference conic. Using the first-order solution given by Equation (14), with the constants of integration determined by Equations (15) for the revolution for which we desire the higher-order solution, the 
Picard iteration yields the higher-orders and more accurate constants of integration by continual application of Equations (15). Since the accuracy of the constants depends on the accuracy of the solution of the previous or subsequent revolution we must begin at the epoch and proceed backward or forward in time.

The value of $c^{\prime}$ (or $y$ ) during the Picard iteration remains fixed for each revolution or interval in $u$ since it is defined here as the mean anomaly of the planet at perihelion passage of the comet on the reference ellipse.

For iterates past the first, the motion of the perturbing planets may be allowed to deviate from the elliptic motion assumed in the first-order solution. Since the special values for the harmonic analysis of $\varphi_{n}, n>0$, depend on numerical values of $\mathbf{r}_{j}^{\prime}$ for each iteration, values of $\mathbf{r}_{j}^{\prime}$ not derived from elliptic motion may be used for $n>0$. This relaxation is admitted due to the fact that validity of the higher-order solutions exists only for a definite interval of time. Also, for the higher iterates, the variable $y$ may be given its actual numerical value appropriate to the interval under consideration. This allows the expansions to collapse from double series in $x$ and $y$ to single series in $x$ for the higher orders.

For the first-order solution to be valid for all intervals, the double series and conic motion for the planets must be retained. It is, of course, preferable to use mean conics for both the comet and the planets.

It may be noted that for the higher-order iterates, the interval may include more than one interval of the first-order solution. Including more intervals would necessitate more iterates to the desired convergence, but the added efficiency of the calculations may prove advantageous. Also, it is known that in the Picard iteration using Chebyshev polynomials one may begin in the first iterates with a fewer number of terms in the series and increase the number of terms in the series for the higher iterates as convergence is approached (see, for example, Norton, 1964). Hence, the first-order solution may contain far fewer terms than the higher-order solutions. Another comment, noted by Broucke (1969), is that the solution may be modified or corrected in only one or two additional iterations without having to do the complete numerical integration over again.

If the comet encounters actual close approaches or if the converged solution begins to deviate substantially from the conic and first-order reference solution, one must then perform a rectification of the reference solution. The conic and first-order solution must be recalculated. The process is analogous to rectification in Encke's method of special perturbations. But rectification will not be required as often as it would be for Encke's method since the present method incorporates a more accurate reference solution. Also, the perturbations about this reference solution will generally be much smaller than perturbations about conic motion in the same interval of time.

\section{Acknowledgments}

I wish to thank P. Herget of the University of Cincinnati, and V. Szebehely, G. Giacaglia, and T. Feagin of the University of Texas at Austin, for several suggestions and for many helpful discussions. 
The financial support of the National Science Foundation, Grant No. GP-17369, and the Office of Naval Research, Grant No. N00014-67-A-0126-0007, is gratefully acknowledged.

\section{References}

Broucke, R.: 1969, Celes. Mech. 1, 110.

Carpenter, L.: 1966, NASA TN D-3168.

Clenshaw, D. W. and Norton, H. J.: 1963, Comput. J. 6, 88.

Fox, L. and Parker, I. B.: 1968, Chebyshev Polynomials in Numerical Analysis, Oxford University Press, London.

Gyldén, H.: 1870, Bull. Acad. Imp. Sci. St. Petersb. 14, 195.

Hansen, P.: 1856, Suppl. Compt. Rend. Acad. Sci. Paris 1, 121.

Hansen, P.: 1857, Abh. Königl. Sächs. Ges. Wiss. Math.-Phys. Kl. 3, 41.

Herget, P.: 1948, The Computation of Orbits, Cincinnati.

Ince, E. L.: 1956, Ordinary Differential Equations, Dover Publ., New York.

Nacozy, P.: 1969, Astron. J. 74, 544.

Norton, H. J.: 1964, Comput. J. 7, 76.

\section{Discussion}

G. N. Duboshin: Did you perform any comparison with numerical integration?

P. E. Nacozy: I have not compared the method with solutions obtained by numerical integration. But it appears that the method will provide solutions with more uniform and definitive error bounds due to the properties of Chebyshev polynomial series. It also appears that some advantages may result by using a reference solution that includes the first-order perturbations, as well as the conic motion. 